\title{
Anti-Aging in ultrastable metallic glasses
}

\author{
Martin Lüttich, ${ }^{1,2, *}$ Valentina M. Giordano, ${ }^{3}$ Sylvie Le Floch,${ }^{3}$ Eloi Pineda, ${ }^{4}$ \\ Federico Zontone, ${ }^{2}$ Yuansu Luo, ${ }^{1}$ Konrad Samwer, ${ }^{1}$ and Beatrice Ruta ${ }^{3,2, \dagger}$ \\ ${ }^{1}$ I. Physikalisches Institut, Universität Göttingen, \\ Friedrich-Hund-Platz 1, 37077 Göttingen, Germany \\ ${ }^{2}$ ESRF - The European Synchrotron, CS 40220, 38043 Grenoble Cedex 9, France \\ ${ }^{3}$ Univ Lyon, Université Claude Bernard Lyon 1, CNRS, \\ Institut Lumière Matière, F-69622, Villeurbanne, France \\ ${ }^{4}$ Department of Physics, Universitat Politècnica de Catalunya-BarcelonaTech, Esteve Terradas 8, Castelldefels 08860, Spain
}

(Dated: November 30, 2017)

\begin{abstract}
As ultrastable metallic glasses (UMGs) are promising candidates to solve the stability issues of conventional metallic glasses, their study is of exceptional interest. By means of x-ray photon correlation spectroscopy, we have investigated the stability of UMGs at the atomic level. We find a clear signature of ultrastability at the atomic level which results in slower relaxation dynamics of UMGs with respect to conventional (rapidly quenched) metallic glasses, and in a peculiar acceleration of the dynamics by near $T_{\mathrm{g}}$ annealing. This surprising phenomenon, called here Anti-Aging, can be understood in the framework of the potential energy landscape. For all samples, the structural relaxation process can be described with a highly compressed shape of the density fluctuations, unaffected by thermal treatments and regardless of the ultrastability of the glass.
\end{abstract}

Due to their extraordinary thermodynamic and kinetic stability, ultrastable glasses (USGs) form a new and promising family of glasses. This stability is a direct consequence of their fabrication process. Initially discovered in 2007 by Swallen et al. [1], USGs have gained interest in the scientific community. In particular, USGs usually exhibit a significant increase in the glass transition temperature $\left(T_{\mathrm{g}}\right)$ and a decrease in the fictive temperature $\left(T_{\mathrm{f}}\right)$ by several tens of Kelvin, as well as an increased density with respect to standard quenched glasses [1-4]. Besides, organic USGs show a decreased enthalpy which seems to be not the case for their metallic counterpart $[5,6]$. With respect to conventional glasses, USGs are considered to be in a lower state in the potential energy landscape (PEL) [7-9], which is reached thanks to enhanced atomic mobility during fabrication process [10]. This deep position in the PEL is not accessible on practical timescales through classical methods as for example long annealing $[3,11]$. With all these properties, USGs are an important building block for many applications like for pharmaceuticals or technical purposes - profiting from longer preservation of the amorphous state and its advantages $[12,13]$.

In order to achieve these enhanced properties, USGs are produced by physical vapor deposition while the substrate is kept at a temperature between $0.7 T_{\mathrm{g}}$ and $0.9 T_{\mathrm{g}}$ and the deposition rate is comparatively low (of the order of $1 \mathrm{~nm} \mathrm{~s}^{-1}$ ) [1, 11, 14]. With these two fabrication parameters, vapor deposition of amorphous materials enhances the surface mobility. Swallen et al. showed that the surface mobility increases by more than six orders of magnitude in molecular glasses, compared to their liquidquenched counterparts [11]. This seems to be the key for producing USGs, as enhanced surface mobility together with a low quenching rate allows the molecules to have an increased amount of time for configuration sampling.

Following the recipe of the first USGs from organic systems, recently $Y u$ et al. reported on the fabrication of an ultrastable metallic glass (UMG) [5]. By means of vapor deposition, they produced a $\mathrm{Zr}_{65} \mathrm{Cu}_{27.5} \mathrm{Al}_{7.5} \mathrm{UMG}$ at a substrate temperature $T_{\text {sub }} \approx 0.8 T_{\mathrm{g}}$ (with respect to $T_{\mathrm{g}}$ of the conventionally produced (rapidly quenched) MG of the same composition) and found an increase $\Delta T_{\mathrm{g}}=+11 \mathrm{~K}$ and ca. $8 \%$ increase of the indentation modulus, compared to the conventional MG. The fabrication of the first UMG is promising as it could enable some of the technical limitations of MGs to be overcome. Conventional MGs are indeed considered ideal candidates for technological usage due to their improved properties like elasticity, hardness and chemical corrosion resistance, compared to their (poly-)crystalline counterparts. MGs suffer however from the spontaneous evolution of their properties due to underlying relaxation processes occurring in the material during usage [13]. This phenomenon is known as physical aging (in the following simply "aging") and is reported in simulations $[15,16]$ and experiments [17-19] in all kind of glasses and is particularly pronounced in MGs [20, 21]. Macroscopic studies in ultrastable organic glasses indicate the absence of physical aging in these systems [22], but no direct information on the microscopic processes have been reported so far.

In this Letter, we present an investigation of the microscopic mechanisms governing the structural relaxation in free standing films of ultrastable metallic $\mathrm{Cu}_{50} \mathrm{Zr}_{50}$ glasses by looking at their collective atomic motion. For this purpose, we use x-ray photon correlation spectroscopy (XPCS) to explore the evolution of density fluctuations at the atomic length scale [23] over timescales from $1 \mathrm{~s}$ to $10^{4} \mathrm{~s}$.

Up to now, XPCS has been used to study the dy- 
namical properties of conventional (rapidly quenched) MGs [24], while it has never been used for UMGs. Here we find that UMGs display structural rearrangements on a length scale of a few atomic distances with a characteristic time of the order of $10^{3} \mathrm{~s}$, even hundreds of degrees below $T_{\mathrm{g}}$. Similar results were reported for conventional MGs [25, 26], strengthening the idea of the existence of structural relaxations in glasses on a length scale of a few Angström, independently of their macroscopic behavior, as confirmed also by measurements of the $\beta$-relaxation in MGs [27].

We produced vapor deposited $\mathrm{Cu}_{50} \mathrm{Zr}_{50}$ by magnetron sputtering onto a polished $\mathrm{NaCl}$ substrate, with $T_{\mathrm{sub}}=0.89(1) T_{\mathrm{g}}$. With this method we obtained a free standing UMG foil, $20 \times 30 \mathrm{~mm}$ and ca. $5 \mu \mathrm{m}$ thick (details in ref. [5]). The UMG has been characterized by $\mathrm{x}$-ray diffraction in order to confirm the amorphous structure. We studied four samples of $\mathrm{Cu}_{50} \mathrm{Zr}_{50}$ : an asproduced UMG ("UMG ${ }_{\mathrm{ap}}$ "); two annealed at $T_{\mathrm{a}}=650 \mathrm{~K}$

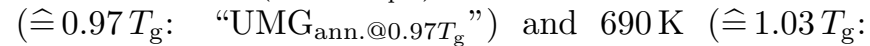
"UMG $\mathrm{Unn}_{\text {an. @1.03Tg }}$ "), respectively; and one conventional (rapidly quenched) ribbon of the same composition. The annealing was done by heating an $\mathrm{UMG}_{\mathrm{ap}}$ with a rate of $1.17 \mathrm{~K} \mathrm{~s}^{-1}$ and holding the annealing temperature $T_{\mathrm{a}}$ for $1 \mathrm{~min}$ before cooling to room temperature with the same rate. The choice of the annealing temperatures $T_{\mathrm{a}}=(1 \pm 0.3) T_{\mathrm{g}}$ was dictated by the necessity to keep the dynamics in the experimental window available for the XPCS technique and prevent also possible crystallization in the supercooled liquid. Two further samples, deposited at $T_{\text {sub }}=0.80(1) T_{\mathrm{g}}$ and $0.18(2) T_{\mathrm{g}}$, were also produced and studied.

XPCS measurements were performed at beamline ID10 at ESRF (France) by using a $10 \times 7 \mu \mathrm{m}(\mathrm{h} \times \mathrm{v})$ partially coherent beam, provided by an undulator source at $8.1 \mathrm{keV}$ photon energy. The samples were measured in transmission in wide angle geometry at a wave vector $q \approx 2.7 \AA^{-1}$ corresponding to the first sharp diffraction peak (FSDP), which is a manifestation of the average inter-particle order (distance) of the amorphous system. This is the relevant length scale for the microscopic configuration of the system. For this reason, structural relaxations are evaluated both experimentally (XPCS, quasi-elastic neutron scattering) and theoretically (molecular dynamics simulations) at the FSDP. Series of speckle patterns were collected by an Andor iKon-M CCD, placed $70 \mathrm{~cm}$ downstream the sample. The samples were kept under vacuum ( $p \lesssim 10^{-4} \mathrm{mbar}$ ) in a home made furnace with a temperature stability better than $\pm 0.05 \mathrm{~K}$. Intensity autocorrelation functions $g^{(2)}(q, t)$ were calculated using the event correlator technique and dropletizing schemes [28]. As explained in ref. [29], $g^{(2)}(q, t)$ can be related to density fluctuations, thus the dynamics, through the Siegert relation (Eq. 1)
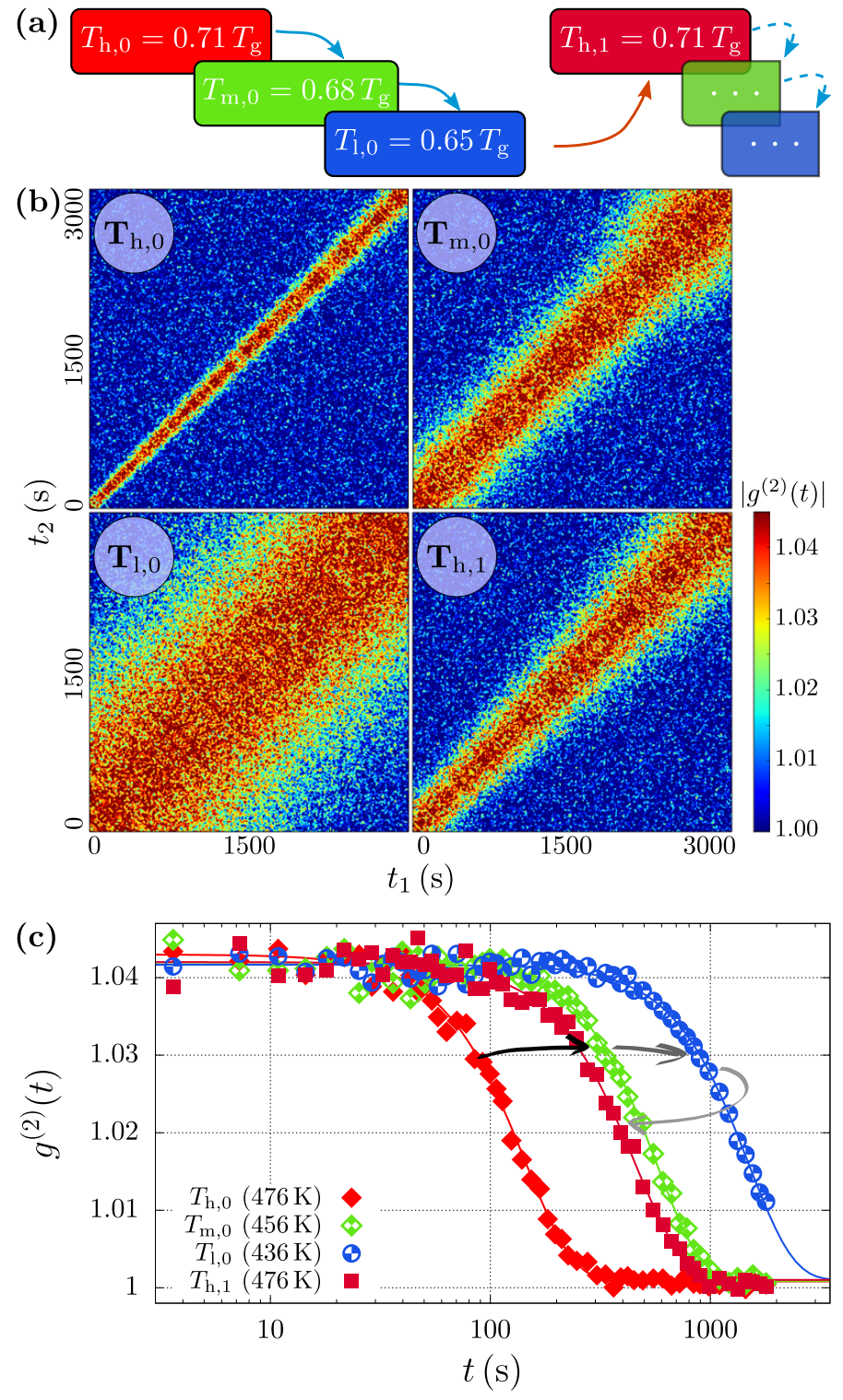

FIG. 1. (a) Scheme of applied temperature cycling. Relative temperatures are related to $T_{\mathrm{g}}$ of conventional $\mathrm{Cu}_{50} \mathrm{Zr}_{50}$ MG. (b) TTCFs of $\mathrm{UMG}_{\mathrm{ap}}$ along first cycle in the $T$-protocol. Changes in the dynamics are signalled by different widths of the reddish area along the main diagonal. All TTFCs are equally scaled. (c) Corresponding $g^{(2)}(q, t)$, obtained by timeaveraging over each TTCF from panel (b).

$$
g^{(2)}(q, t)=1+B(q)|f(q, t)|^{2}
$$

where $\mathrm{B}(\mathrm{q})$ is a setup-dependent parameter and $f(q, t)$ is the intermediate scattering function [24, 29]. Hence, $g^{(2)}(q, t)$ represents a measure of the temporal relaxation of a given microscopic configuration on the spatial scale $2 \pi / q \approx 2-3 \AA$. Its decay time corresponds to the time the system takes to lose memory of its initial configuration and is therefore representative for the dynamics at the probed length scale. 
Data were collected by performing temperature cycles in the glassy state as depicted in figure 1(a). All samples were heated up to the initial high temperature $T_{\mathrm{h}, 0}=476 \mathrm{~K}\left(\widehat{=} 0.71 T_{\mathrm{g}}\right)$. After the first isothermal measurement, the temperature is decreased by $20 \mathrm{~K}$ to a middle value $T_{\mathrm{m}, 0}=456 \mathrm{~K}\left(\widehat{=} 0.68 T_{\mathrm{g}}\right)$ and then lowered to $T_{1,0}=436 \mathrm{~K}\left(\widehat{=} 0.65 T_{\mathrm{g}}\right)$ before being again increased to $T_{\mathrm{h}, 1} \equiv T_{\mathrm{h}, 0}$. This cycle was repeated several times, iteration number $i$ in $T_{\mathrm{h}, i}$ corresponds to the iteration of the cycling. Temperature changes were performed with a heating/cooling rate of $1.17 \mathrm{~K} \mathrm{~s}^{-1}$ while each isotherm was kept for ca. $3.6 \times 10^{3} \mathrm{~s}$.

Figure 1 (b) shows the two-time correlation functions (TTCFs) for $\mathrm{UMG}_{\mathrm{ap}}$, measured at different temperatures $T$. The TTCFs depict the evolution of intensity fluctuations of the captured speckle patterns during the isotherms at the first four steps of the $T$-protocol from high to low values and back $\left(T_{\mathrm{h}, 0} \rightarrow T_{\mathrm{m}, 0} \rightarrow T_{\mathrm{l}, 0} \rightarrow T_{\mathrm{h}, 1}\right)$. In this representation, the experimental time, i.e. the waiting time $t_{\mathrm{w}}$, elapses from the beginning of each isothermal measurement and evolves along the main diagonal. The amplitude profile (color coded) determines the time resolved dynamics; in particular the width of the high-correlation (reddish) area is directly proportional to the relaxation time $[30,31]$.

The relaxation dynamics changes with temperature as the main diagonal is broader at lower temperatures. After re-heating to the initial value (to $T_{\mathrm{h}, 1} \equiv T_{\mathrm{h}, 0}$ ), the sample clearly shows slower relaxation than at $T_{\mathrm{h}, 0}$. We observe also a weak temporal aging during high$T$ isotherms, where the width of the intensity profile along the main diagonal slightly increases with time. This effect is only present in $\mathrm{UMG}_{\mathrm{ap}}$ and is likely due to some residual stresses in the as-prepared material, which then disappears after a few temperature iterations (see also supplemental material fig. 1 and 2). In order to get quantitative information, we average each TTCF along the $t_{\mathrm{w}}$-axis. The resulting $g^{(2)}(q, t)$ function can be fitted by the Kohlrausch-Williams-Watts expression (KWW, $[32,33]) g^{(2)}(q, t)=1+A \exp \left[-2(t / \tau)^{\beta}\right]$, from which we get three parameters: the structural relaxation time $\tau$, the shape parameter $\beta$ and the contrast $A$. Figure 1 (c) shows the $g^{(2)}(q, t)$ function corresponding to the first $T$-cycle reported in figure $1(\mathrm{~b})$. After a small $T$ decrease of $40 \mathrm{~K}\left(T_{\mathrm{h}}\right.$ to $\left.T_{1}\right)$, the $g^{(2)}(t)$ curve shifts by more than one order of magnitude towards longer time scales. This process is not fully reversible. After re-heating to $T_{\mathrm{h}, 1}$; the data shift back to shorter times without overlapping with the initial curve, hence the $T$-protocol induces some aging. It is important to stress that $g^{(2)}(q, t)$ exhibits a full decorrelation at all $T_{j, i}$. This is striking as one would expect to observe rather slow dynamics in UMGs, with relaxation times much larger that the probed time interval. This surprising relatively fast collective motion is likely to be a consequence of the probed atomic length scale, which is not representative for the
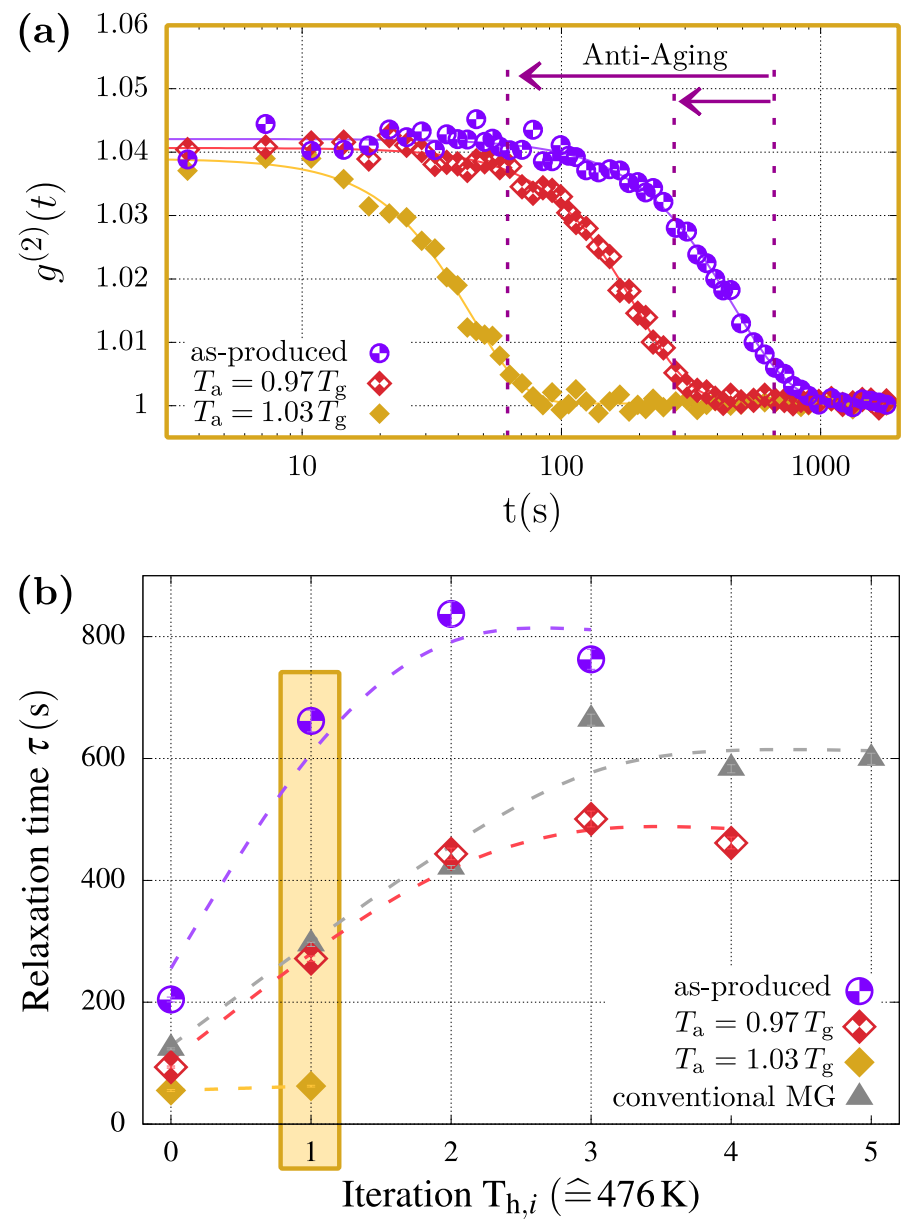

FIG. 2. (a) Intensity autocorrelation functions of $\mathrm{UMG}_{\mathrm{ap}}$

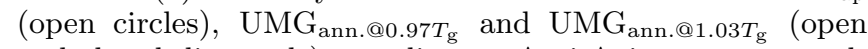
and closed diamonds) revealing an Anti-Aging upon annealing. These $g^{(2)}(t)$ functions correspond to the first temperature iteration $\left(T_{\mathrm{h}, 1} \widehat{=} 476 \mathrm{~K}\right)$. Solid lines are best fits of the KWW expression. Vertical dashed lines indicate the structural relaxation time. (b) Evolution of $\tau$ of $\mathrm{UMG}_{\mathrm{ap}}$ (open circles), pre-annealed UMGs (open and closed diamonds) and conventionally produced MG (triangles) as a function of hightemperature iterations $T_{\mathrm{h}, i}$ along the applied $T$-protocol. The yellow box highlights the data corresponding to panel (a). Dashed lines are a guide to the eye. Errorbars are within the symbol size.

macroscopic picture [23]. The here observed decorrelation is associated to rearrangements on a spatial scale of a few Ångström. Similar fast relaxation times were also observed in $\mathrm{UMG}_{\mathrm{ann} . @ 0.97 T_{\mathrm{g}}}$ and $\mathrm{UMG}_{\mathrm{ann} . @ 1.03 T_{\mathrm{g}}}$.

We also find clear signature of ultrastability at the atomic level which results in higher stability of the $\mathrm{UMG}_{\mathrm{ap}}$ with respect to the other glasses. This is shown in figure 2 (a) where we compare the correlation function measured at $T_{\mathrm{h}, 1}$ for $\mathrm{UMG}_{\mathrm{ap}}$ with those measured in ultrastable glasses which were previously annealed below and above $T_{\mathrm{g}}\left(\mathrm{UMG}_{\mathrm{ann} . @ 0.97 T_{\mathrm{g}}}\right.$ and $\mathrm{UMG}_{\mathrm{ann} . @ 1.03 T_{\mathrm{g}}}$, respectively). 
Upon annealing, we do not find the typical behavior of slowing down dynamics due to relaxation, but the opposite: an acceleration of the dynamics towards shorter time scales. We call this anomalous behavior Anti-Aging as it evolves towards the opposite direction as it is observed in conventional MGs, where pre-annealing is often used to improve their stability $[2,6,26,34,35]$. The enhanced stability of $\mathrm{UMG}_{\text {ap }}$ persists at all investigated temperatures and during all $T$-cycling. The relaxation times $\tau$ at $T_{\mathrm{h}, i}$ of all three UMGs (as produced and differently pre-annealed) and of the conventional MG are merged in figure $2(\mathrm{~b})$. $\mathrm{UMG}_{\mathrm{ap}}$ has the largest relaxation times at all iterations (very slow process), a signature of the improved stability obtained by the physical vapour deposition. The Anti-Aging is signalled by the decrease of $\tau$ in the annealed samples ( $\mathrm{UMG}_{\mathrm{ann} . @ 0.97 T_{\mathrm{g}}}$ and $\mathrm{UMG}_{\mathrm{ann} . @ 1.03 T_{\mathrm{g}}}$ ). The dynamics of $\mathrm{UMG}_{\mathrm{ap}}$ is even slower than that of the conventional MG (triangles), whose relaxation times are instead similar to the UMG pre-annealed below $T_{\mathrm{g}}\left(\mathrm{UMG}_{\mathrm{ann} . @ 0.97 T_{\mathrm{g}}}\right)$.

Besides ultrastability, XPCS measurements allow us to study also the temporal evolution of the dynamics due to physical aging. $\mathrm{UMG}_{\mathrm{ap}}, \mathrm{UMG}_{\mathrm{ann} . @ 0.97 T_{\mathrm{g}}}$ and the conventional MG exhibit a history dependence where $\tau$ increases with the number of iterations $i$ and then becomes constant for $i \geq 2$. The same was found at $T_{\mathrm{m}, i}$ and $T_{1, i}$. Differently, $\mathrm{UMG}_{\text {ann.@1.03T }} T$-cycling reveals complete reversible dynamics at all iterations and temperatures (see supplemental material fig. 4). The dependence of $\tau$ on the iterations at the beginning of the applied protocol can be associated to aging induced by $T$-cycling (Debye-Grüneisen effect), while after a few iterations $\mathrm{UMG}_{\mathrm{ap}}, \mathrm{UMG}_{\mathrm{ann} . @ 0.97 T_{\mathrm{g}}}$ and the conventional MG are trapped in a metastable state where the dynamics is more localized [36]. The existence of aging and stationary regimes has been reported in many XPCS measurements on MGs [24, 37] and it has been attributed to structural rearrangements, driven by density changes (aging regime) and increasing correlation length at the medium range order (stationary regime) [38]. In those works, aging occurs through spontaneous temporal evolutions, while here the majority of the data correspond to stationary dynamics within the probed time interval, as depicted in figure 1 (b) (compare also supplemental material fig. 1 and 2 ). It is important to note that the regime where $\tau$ is independent on the iterations does not correspond to an equilibrium situation. As shown in recent works, aging is likely to continue through intermittent jumps between stationary regimes [24, 25].

The unique Anti-Aging properties of UMGs with respect to conventional MGs can be better understood looking at figure 3 where we report the relaxation times measured at $T_{\mathrm{h}, 1}$ corresponding to the data in figure 2 (b) (open diamonds), together with those measured at the same temperature in an as-prepared conventional MG and in a second $\mathrm{MG}$ pre-annealed at $0.97 T_{\mathrm{g}}$ (trian-

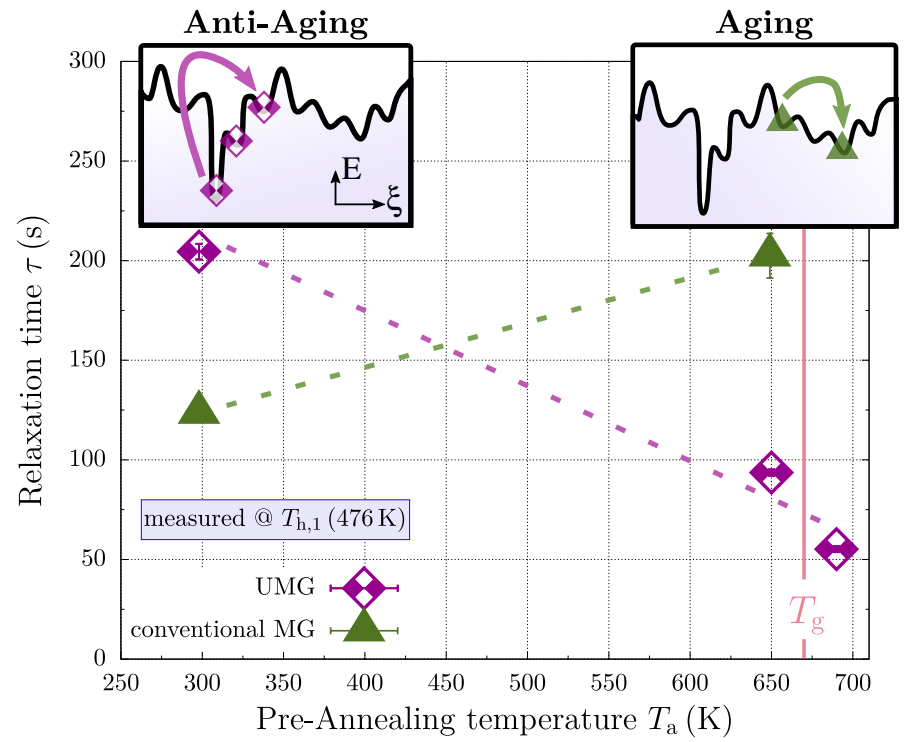

FIG. 3. Effect of pre-annealing temperature $T_{\mathrm{a}}$ on $\tau$ for conventional $\mathrm{Cu}_{50} \mathrm{Zr}_{50}$ MG (triangles) and UMG (open diamonds), measured at $T_{\mathrm{h}, 1}(\widehat{=} 476 \mathrm{~K})$. The data at $298 \mathrm{~K}$ correspond to as-prepared samples. The vertical line depicts $T_{\mathrm{g}}$ of the conventional MG. The Anti-Aging of the UMG is indicated by the $T_{\mathrm{a}}$-dependence which evolves inverse as normal aging. Dashed lines are a guide to the eye. Two insets depict a scheme of the data interpretation within the framework of the PEL.

gles). In the MGs, we see the typical aging with an increase of $\tau$ upon annealing. We may understand this behavior within the framework of the PEL $[8,9]$. Conventional MGs are known to be located in an energetically high basin in the PEL and thus can relax down by annealing, leading towards increased relaxation times $[2,4,10,11,14]$. As high-substrate-temperature vapor deposited glasses are supposed to be trapped in a very low basin of the PEL directly by the fabrication process, they should have long relaxation times, accordingly. Upon annealing near $T_{\mathrm{g}}$ their relaxation time decreases. This suggests that the energy introduced by temperature changes boosts the system in to a PEL basin located higher than the initial one.

Another signature of the ultrastability of $\mathrm{UMG}_{\mathrm{ap}}$ is given by the dependence of $\tau$ on $T_{\text {sub }}$ used during vapour deposition. This is shown in figure 4 where we report $\tau$ at the initial temperature step $T_{\mathrm{h}, 0}$ for vapor deposited $\mathrm{Cu}_{50} \mathrm{Zr}_{50}$ produced at different substrate temperatures. We find that $\tau$ strongly increases for $T_{\text {sub }}=0.80 T_{\mathrm{g}}$ and $0.89 T_{\mathrm{g}}$, confirming the ultrastability of these glasses. Similar results have been found for $\mathrm{Zr}_{65} \mathrm{Cu}_{27.5} \mathrm{Al}_{7.5}$ [5].

Despite the observed ultrastability, the shape parameter $\beta$ of the $g^{(2)}(t)$ functions remains the same at all probed temperatures and for all samples (see supplemental material fig. 3). In all cases the curves decay in a highly compressed manner with $\beta=2.0(2)$, similarly to what is reported for other metallic and non-metallic 


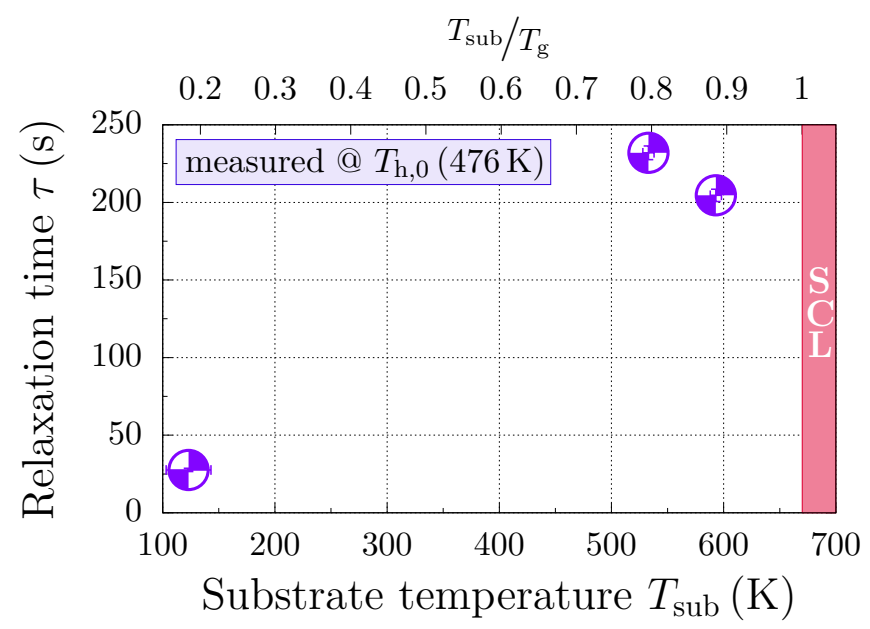

FIG. 4. Structural relaxation times measured at $T_{\mathrm{h}, 0}$ for three $\mathrm{Cu}_{50} \mathrm{Zr}_{50}$ films, prepared at different $T_{\text {sub }}$. Errorbars are within the symbol size. The red box indicates the supercooled liquid area with respect to $T_{\mathrm{g}}$ of the conventional MG.

glassy systems [25, 37, 39-41]. This is astonishing as compressed correlation functions are often associated with internal stresses $[42,43]$ and ultrastability is usually related to low values of stress. In our case, $\beta$ is not only very large but completely unaffected by annealing or sample preparation. This means that elastic frustrations - responsible for stress dominated dynamics - are not involved in the process leading to ultrastability, and the stresses related to a high $\beta$ are likely intrinsic to the out-of-equilibrium nature of the glassy state in metallic systems.

In conclusion, we have presented a detailed investigation of the relaxation processes occurring at the atomic level in UMGs. Differing from conventional glasses, asproduced UMGs are found to be the most stable glasses, exhibiting a remarkable Anti-Aging when pre-annealed at temperatures around $T_{\mathrm{g}}$. This effect does not occur in conventional MGs, adding another feature to the list of the unique properties of ultrastable glasses.

We acknowledge Y. Chushkin (ESRF) for providing software to analyze the raw data, K. L'Hoste and H. Vitoux (ESRF) for technical support with the XPCS experiments, ESRF for providing beamtime and K.S. acknowledges H.-B. Yu (Huazhong Univ. China) for fruitful discussions.

* mluetti1@gwdg.de

† beatrice.ruta@univ-lyon1.fr

[1] S. F. Swallen, K. L. Kearns, M. K. Mapes, Y. S. Kim, R. J. McMahon, M. D. Ediger, T. Wu, L. Yu, and S. Satija, Science 315, 353 (2007).

[2] K. L. Kearns, S. F. Swallen, M. D. Ediger, T. Wu, and L. Yu, J. Chem. Phys. 127, 154702 (2007).

[3] S. F. Swallen, K. L. Kearns, S. Satija, K. Traynor, R. J.
McMahon, and M. D. Ediger, J. Chem. Phys. 128, 214514 (2008).

[4] S. Singh, M. D. Ediger, and J. J. de Pablo, Nat. Mater. 12, 139 (2013).

[5] H.-B. Yu, Y. Luo, and K. Samwer, Adv. Mater. 25, 5904 (2013).

[6] R. J. Xue, L. Z. Zhao, C. L. Shi, T. Ma, X. K. Xi, M. Gao, P. W. Zhu, P. Wen, X. H. Yu, C. Q. Jin, M. X. Pan, W. H. Wang, and H. Y. Bai, Appl. Phys. Lett. 109, 221904 (2016).

[7] F. H. Stillinger, Science 267, 1935 (1995).

[8] P. G. Debenedetti and F. H. Stillinger, Nature 410, 259 (2001).

[9] Z. Raza, B. Alling, and I. A. Abrikosov, J. Phys. Condens. Matter 27, 293201 (2015).

[10] G. Parisi and F. Sciortino, Nat. Mater. 12, 94 (2013).

[11] K. L. Kearns, S. F. Swallen, M. D. Ediger, T. Wu, Y. Sun, and L. Yu, J. Phys. Chem. B 112, 4934 (2008).

[12] B. C. Hancock and M. Parks, Pharm. Res. 17, 397 (2000).

[13] W. H. Wang, Prog. Mater. Sci. 57, 487 (2012).

[14] K. J. Dawson, K. L. Kearns, M. D. Ediger, M. J. Sacchetti, and G. D. Zografi, J. Phys. Chem. B 113, 2422 (2009).

[15] W. Kob and J.-L. Barrat, Phys. Rev. Lett. 78, 4581 (1997).

[16] J. Rottler and M. O. Robbins, Phys. Rev. Lett. 95, 225504 (2005).

[17] L. C. E. Struik, Physical aging in amorphous polymers and other materials, Ph.D. thesis, Delft University of Technology (1977).

[18] I. M. Hodge, Science 267, 1945 (1995).

[19] L. Cipelletti, L. Ramos, S. Manley, E. Pitard, D. A. Weitz, E. E. Pashkovski, and M. Johansson, Faraday Discuss. 123, 237 (2003).

[20] J. Hachenberg, D. Bedorf, K. Samwer, R. Richert, A. Kahl, M. D. Demetriou, and W. L. Johnson, Appl. Phys. Lett. 92, 131911 (2008).

[21] F. Zhai, E. Pineda, B. Ruta, M. Gonzalez-Silveira, and D. Crespo, J. Alloys Compd. 615, S9 (2014).

[22] E. Leon-Gutierrez, G. Garcia, A. F. Lopeandia, M. T. Clavaguera-Mora, and J. Rodriguez-Viejo, J. Phys. Chem. Lett. 1, 341 (2010).

[23] B. Ruta, Y. Chushkin, G. Monaco, L. Cipelletti, E. Pineda, P. Bruna, V. M. Giordano, and M. GonzalezSilveira, Phys. Rev. Lett. 109, 165701 (2012).

[24] B. Ruta, E. Pineda, and Z. Evenson, J. Phys. Condens. Matter 29, 503002 (2017).

[25] Z. Evenson, B. Ruta, S. Hechler, M. Stolpe, E. Pineda, I. Gallino, and R. Busch, Phys. Rev. Lett. 115, 175701 (2015).

[26] X. Wang, B. Ruta, L. Xiong, D. Zhang, Y. Chushkin, H. Sheng, H. Lou, Q. Cao, and J. Jiang, Acta Mater. 99, $290(2015)$.

[27] H.-B. Yu, W.-H. Wang, and K. Samwer, Mater. Today 16, 183 (2013).

[28] Y. Chushkin, C. Caronna, and a. Madsen, J. Appl. Crystallogr. 45, 807 (2012).

[29] A. Madsen, A. Fluerasu, and B. Ruta, in Synchrotron Light Sources Free. Lasers (Springer International Publishing, Cham, 2015) pp. 1-21.

[30] M. Sutton, K. Laaziri, F. Livet, and F. Bley, Opt. Express 11, 2268 (2003).

[31] O. Bikondoa, J. Appl. Crystallogr. 50, 357 (2017).

[32] F. Kohlrausch, Ann. der Phys. und Chemie 195, 337 
(1863).

[33] G. Williams and D. C. Watts, Trans. Faraday Soc. 66, 80 (1970).

[34] A. J. Kovacs, Fortschritte der Hochpolym. 3, 394 (1964).

[35] R. Richert, K. Duvvuri, and L.-T. Duong, J. Chem. Phys. 118, 1828 (2003).

[36] Y. Fan, T. Iwashita, and T. Egami, Phys. Rev. Lett. 115, 045501 (2015).

[37] B. Ruta, G. Baldi, G. Monaco, and Y. Chushkin, J. Chem. Phys. 138, 054508 (2013).

[38] V. M. Giordano and B. Ruta, Nat. Commun. 7, 10344 (2016).
[39] J.-P. Bouchaud and E. Pitard, Eur. Phys. J. E - Soft Matter 6, 231 (2001).

[40] S. Gokhale, R. Ganapathy, K. H. Nagamanasa, and A. K. Sood, Phys. Rev. Lett. 116, 068305 (2016).

[41] M. Bouzid, J. Colombo, L. V. Barbosa, and E. Del Gado, Nat. Commun. 8, 15846 (2017).

[42] J.-P. Bouchaud, in Anomalous Transp. (Wiley-VCH Verlag GmbH \& Co. KGaA, Weinheim, Germany, 2008) pp. 327-345.

[43] P. Chaudhuri and L. Berthier, Phys. Rev. E 95, 060601 (2017). 